\title{
Acute high dose Of L-Lysine amino acid leads nephrotoxicity and hepatotoxicity in fresh water fish Clarias batrachus
}

\author{
Dhami B. S. 网
}

Received: 26.08.2020

Revised: 14.10.2020

Accepted: 18.11.2020

\begin{abstract}
The present investigation was carried out to find out the effects of high dose of L-lysine on the fish and also an attempt has been made to predict the impact on human health. L-lysine is an essential amino acid which is used in medical practices as a growth promoter of bones especially in infants. Important work on metabolism and functions of amino acids in human health and major diseases has been reported by many workers and evaluated the key role of various amino acids in response to infections. Lysine is not synthesized by body; therefore, it must be taken either by diet or supplementation. Lysine first of all isolated from casein in 1889 and introduced as Lysine hydrochloride in 1955 . The recommended dose of lysine is $12 \mathrm{mg} / \mathrm{kg}$ body weight. Side effects of Lysine in large doses i.e. 10-30gm/day may cause abdominal cramps and diarrhea but renal and hepatic toxicity is not reported in normal person. Amino acids are used to treat end stage renal failure and also in liver failure. Recently lysine is used to treat herpes and as a supplement for diabetic people. In present study the effect of acute high dose $2 \mathrm{mg} / 100 \mathrm{gm}$ body weight of L-lysine in fish shows toxic effects on kidney and liver which is an alarming indication towards use of high dose of Lysine in man. In kidney it caused glomerular dilation of the capillaries, glomerular hemorrhages and cloudy swelling of renal tubules. In liver it leads swelling of hepatocytes, vacuolization, fatty degeneration and central vein dilation. These histopathological changes are supported by marked rise in level of blood urea, serum creatinine, blood sugar and serum cholesterol.
\end{abstract}

Key Words: Clarias, Dilation, Hepatocytes, Hepatotoxicity, L-lysine, Nephrotoxicity

\section{Introduction}

Amino acids are basic to life, there are twenty amino acids, of these ten are essential \& other ten are non-essential. L-lysine is a basic essential amino acid and not synthesized by body, it is synthesized by plants \& bacteria from aspartic acid. Natural sources of Lysine are red meat, chicken, eggs, fish, beans, nuts, soybeans, spirulina etc. It is being used as one of the nutritional supplement in commercially available food products such as Promolan, Hermin, Astymin Forte, Lysiron and many more nutritional forms as tablets and syrups. Lysine supplements are used in medical practices in malnutrition, chronic diseases, renal and liver disorders, chronic gastrointestinal diseases and alcoholism. Lysine is used to treat herpes Venthan and Sanketh (2017). Recently it is used as a new supplement for nutritional support for diabetic people and people at risk of developing diabetes (John, 2018). Role of amino acids in human body related to health and in some major

Author's Address

Department of Zoology Bareilly College Bareilly - 243005 (UP) India

E-mail.: bsdhamimcm@gmail.com diseases described by Munro (1982) and evaluated the key role of various individual amino acids in response to infections. Crim and Munro (1977) evaluated protein and amino acids requirement in relation to formula diet for medical purpose. Medicinal uses of L- Lysine were described by Meenu et al. (2011). Recently University of Maryland Medical Centre reported L-lysine is essential for human health, but our body cannot synthesize Lysine so we have to get it from food or supplements. Due to medicinal uses Lysine used in daily diet as it lowers cholesterol, helps to absorb calcium and also forms collagen but in a recommended dose. Lee and Kim (2019) worked and reported therapeutic effects of amino acids in liver diseases. The toxicity of different amino acids to vital systems of body worked out by many workers like (Solez, 1983; Malis et al., 1984; Recusen et al., 1985; Asanuma et al., 2006). Amino acids are used to treat toxicity of heavy metals like chromium in fish by Bali and Singh (1994) and Dhami (2012). Some literature is also available on lysine Nephrotoxicity taken with antibiotics like neomycin, gentamycin, streptomycin. The toxicity 
of lysine is least worked out due to its dietary uses however some references are available on its toxicity specially on kidney ,if taken in large doses with impaired kidney function. Steven et al. (2007) reported that L-Lysine caused nephritis and impairment in renal proximal tubule. The use of amino acids in treatment of liver cirrhosis, hepatic comma and hepatic encephalopathy is reported by Park et al. (2017). The work on acute lysine toxicity carried out is so important because one must be careful to take lysine as a dietary supplement in large doses.

\section{Material and Methods}

For this study healthy living freshwater teleost fish Clarias batrachus (Linn.) were selected for experimentation. The fish was obtained from local freshwater sources. Animals acclimatized in glass aquaria in laboratory for 10 days before starting the experiment and fed with commercial fish food twice a day. The animals were divided into two groups. Each group of control and experimental consists 10 animals. The experimental group was given intraperitoneal injection of $2 \mathrm{mg} / 100 \mathrm{gm}$ of body weight/ day for 96 hours to study acute toxicity of L-lysine. The acute toxicity of L-lysine is carried out by $\mathrm{LC}_{50}$ value and during this $50 \%$ of animals died due to toxicity. The fish was injected daily in morning after twenty four hours for 96 hours. After 96 hours of acute treatment kidney and liver dissected out and fixed in alcoholic Bouin's fixative for histopathological investigations. Sections of seven micron were cut in rotator microtome. Staining of tissues carried out in delafield's haematoxylin and alcoholic eosin.

Blood samples were also collected from both control and experimental groups. The blood was taken out by severing tail end. Blood collected in a vial containing anticoagulant EDTA. The hematological parameters were determined by standard methods on Photochem-5 colorimeter. Blood urea was estimated by DAM (Diacetylmonoxime) method, blood glucose estimated by" O' Toludine method, serum creatinine was calculated by Alkaline Picrate Method and serum cholesterol determined by Wybenga and Pileggi Method. The blood parameters data was statistically analysed by calculation of t- test value for difference of means.
Calculation of t-Test for difference of means

' $\mathrm{t}$ ' value was calculated as given under :

$$
t=\frac{\bar{x}-\bar{y}}{\sqrt{\frac{\sum(x-\bar{x})^{2}+(y-\bar{y})^{2}}{n_{1}+n_{2}-2}}} \times \sqrt{\frac{n_{1} \times n_{2}}{n_{1}+n_{2}}}
$$

Where $\bar{X}=$ Mean of the control

$\bar{Y}=$ Mean of the experiment

$(x-\bar{x})^{2}=$ Sum of squares of deviation from mean in control

$(y-\bar{y})^{2}=$ Sum of squares of deviation from mean in experiment

$\mathrm{n}_{1}=$ Sample size of control

$\mathrm{n}_{2}=$ Sample size of experiment

The histopathology of kidney and liver slides after acute exposure to high dose of L-lysine was observed under Olympus laboratory binocular microscope with high power. Eyepiece camera was used to take the photographs in the department of pathology LLRM Medical College Meerut.

\section{Results and Discussion}

The hematological parameters observed for blood urea, serum creatinine, blood sugar and serum cholesterol are given in Table 1 while histopathological changes are shown in fig- 1-3. The findings of renal toxicity are supported by a highly significant statistically increase in blood urea from $5.1 \pm 0.2$ in control to $8.4 \pm 0.45 \mathrm{mg} / 100 \mathrm{ml}$ after acute exposure and serum creatinine from $0.6 \pm 0.041$ in control to $3.0 \pm 0.72 \mathrm{mg} / 100 \mathrm{ml}$ after acute treatment. Rise in blood urea and serum creatinine indicates renal dysfunction, these findings are in agreement with Malis et al., (1984), Recusen et al. (1985), Asanuma et al., (2006), Samyuktha and Francois (2019). Most of these workers found nephrotoxicity in animals by giving a mixture of many amino acids and they observed increased glomerular filtration rate. In present study the only amino acid lysine was used. The possible mechanism of renal damage seems to be that high dose of dibasic cationic amino acid L-lysine produces a rapid decrease in renal function with tubular obstructions, and later on a decrease in renal blood flow, this depresses the renal function and hence produced acute nephrotoxicity and renal failure that caused death of the fish. 


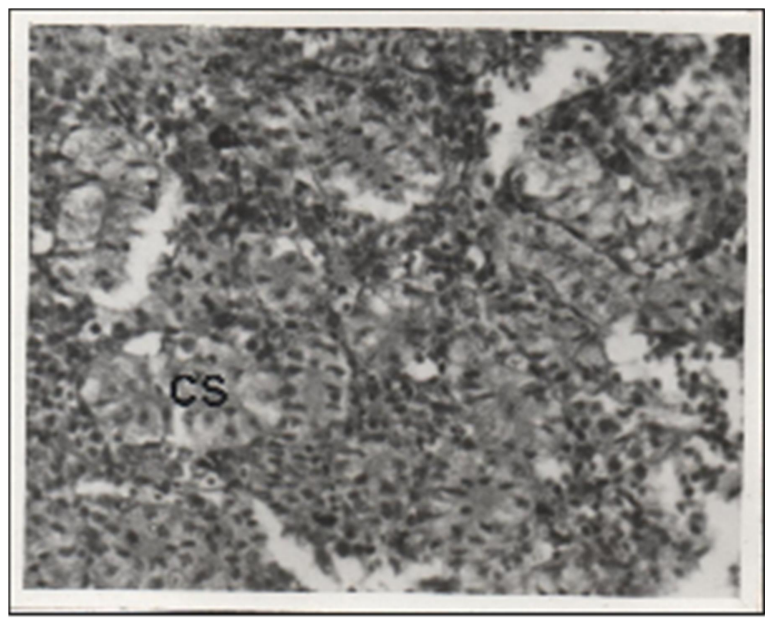

Figure 1. T.S. Kidney(Showing Cloudy Swelling of renal tubules following acute exposure to $\mathrm{L}$ - lysine x400) $C S=$ Cloudy Swelling.

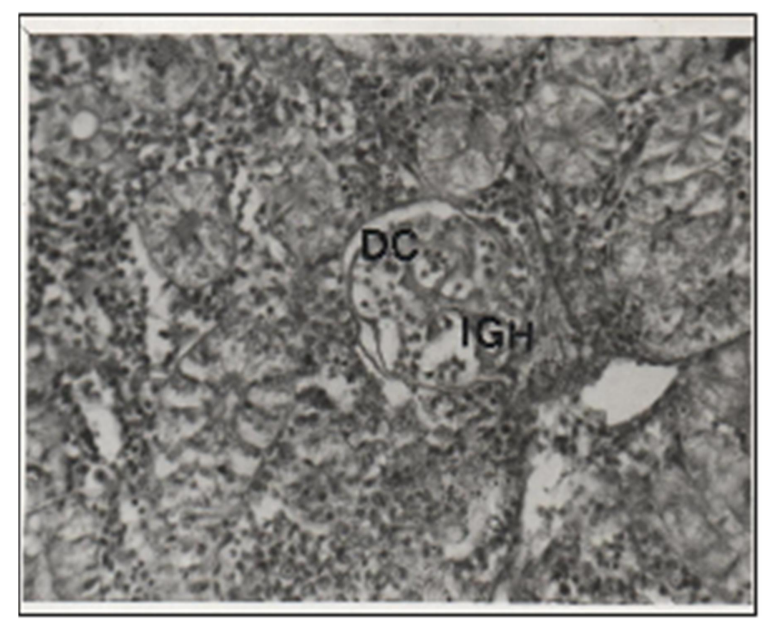

Figure 2. T.S. Kidney( Showing Dilation Glomerulus Capillaries, Interglomerular hemorrhages due to acute exposure to $\mathrm{L}$ - lysine $\mathrm{x}$ 400) $\mathrm{DC}=$ Dilated Capillaries, IGH=Interglomerular Hemorrhages

Arteriosclerosis is a new finding and not found by previous workers in fish and it shows that a high dose of L-lysine may prone to be harmful even in our case because lysine is widely used as a nutritional supplement. However it is known that lysine increases calcium absorption but not reported arteriosclerosis. These studies extend the observation that acute high dose of L-lysine amino acid in fish produced a rapid and permanent decrease in renal function (Steven et al., 2007). It is now reported that a normal intake of Lysine plays an important role in calcium absorption from intestine (Meenu et al., 2011). Present study shows

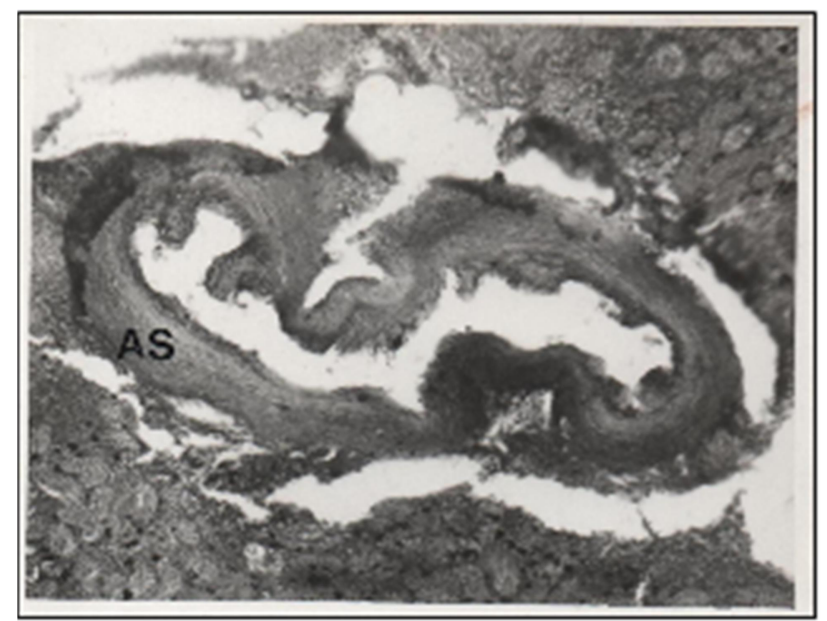

Figure 3. T.S. Kidney(Showing Arterosclerosis following acute exposure L- lysine $x$ 400) $\mathrm{AS}=$ Arteriosclerosis

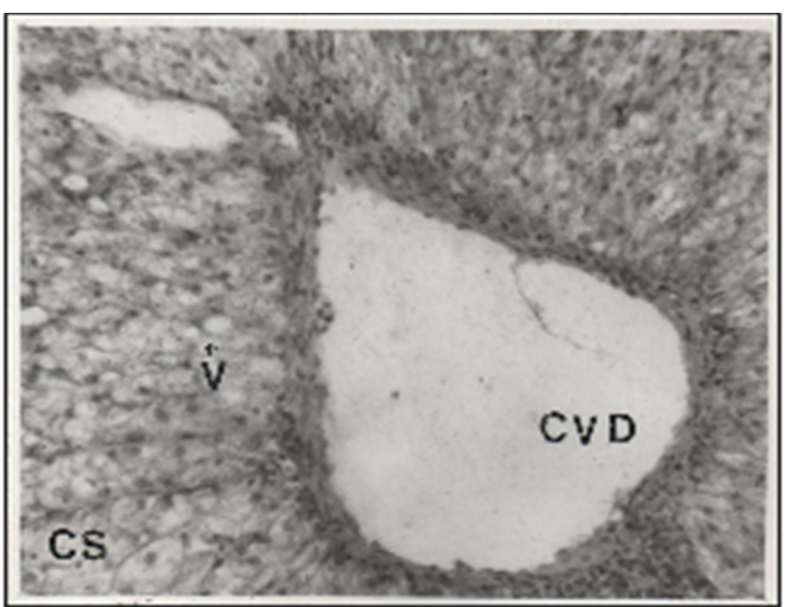

Figure 4. T.S. Liver ( Showing Cloudy Swelling, Vacuolization and Central Vein Dilation after acute exposure to $L$ - lysine $x 400$ ) $C S=C l o u d y$ Swelling, CVS $=$ Central Vein Dilation, $V=$ Vacuolization

that arteriosclerosis occurred due to high dose of Lysine because lysine absorbed large amount of calcium from intestine that caused hypercalcaemia. Nephrotoxicity of Lysine may be related to direct tubular toxicity and to tubular obstruction (Malis et al., 1984). These findings of renal toxicity are in agreement with several researchers (Solez, 1983; Malis et al., 1984; Recusen et al., 1985; Asanuma $\mathrm{K}$ et al., 2006). The acute exposure to L-lysine in experimental fish caused hepatotoxicity such as cloudy swelling of hepatocytes, vacuolization, central vein dialation and fatty degeneration. These hepatic changes are shown in figure-4. All these 
Dhami

Table 1. Hematological parameters observed for blood urea, serum creatinine, blood sugar and serum cholesterol

\begin{tabular}{|l|l|l|l|l|l|}
\hline SN & Blood Parameter & Control & Experimental & $\begin{array}{l}\text { \% alteration from } \\
\text { control }\end{array}$ & t value \\
\hline 1 & Blood urea $(\mathrm{mg} / 100 \mathrm{ml})$ & $5.1 \pm 0.25$ & $8.4 \pm 0.45$ & $64.70 \%$ & $10.32++$ \\
\hline 2 & Serum creatinine $(\mathrm{mg} / 100 \mathrm{ml}$ & $0.6 \pm 0.041$ & $3.0 \pm 0.72$ & $400 \%$ & $39.45++$ \\
\hline 3 & Blood sugar $(\mathrm{mg} / 100 \mathrm{ml})$ & $31 \pm 2.13$ & $60.2 \pm 3.14$ & $94.19 \%$ & $31.62++$ \\
\hline 4 & Serum cholesterol $(\mathrm{mg} / 100 \mathrm{ml})$ & $172 \pm 9.05$ & $180 \pm 7.64$ & $4.65 \%$ & 2.60 \\
\hline
\end{tabular}
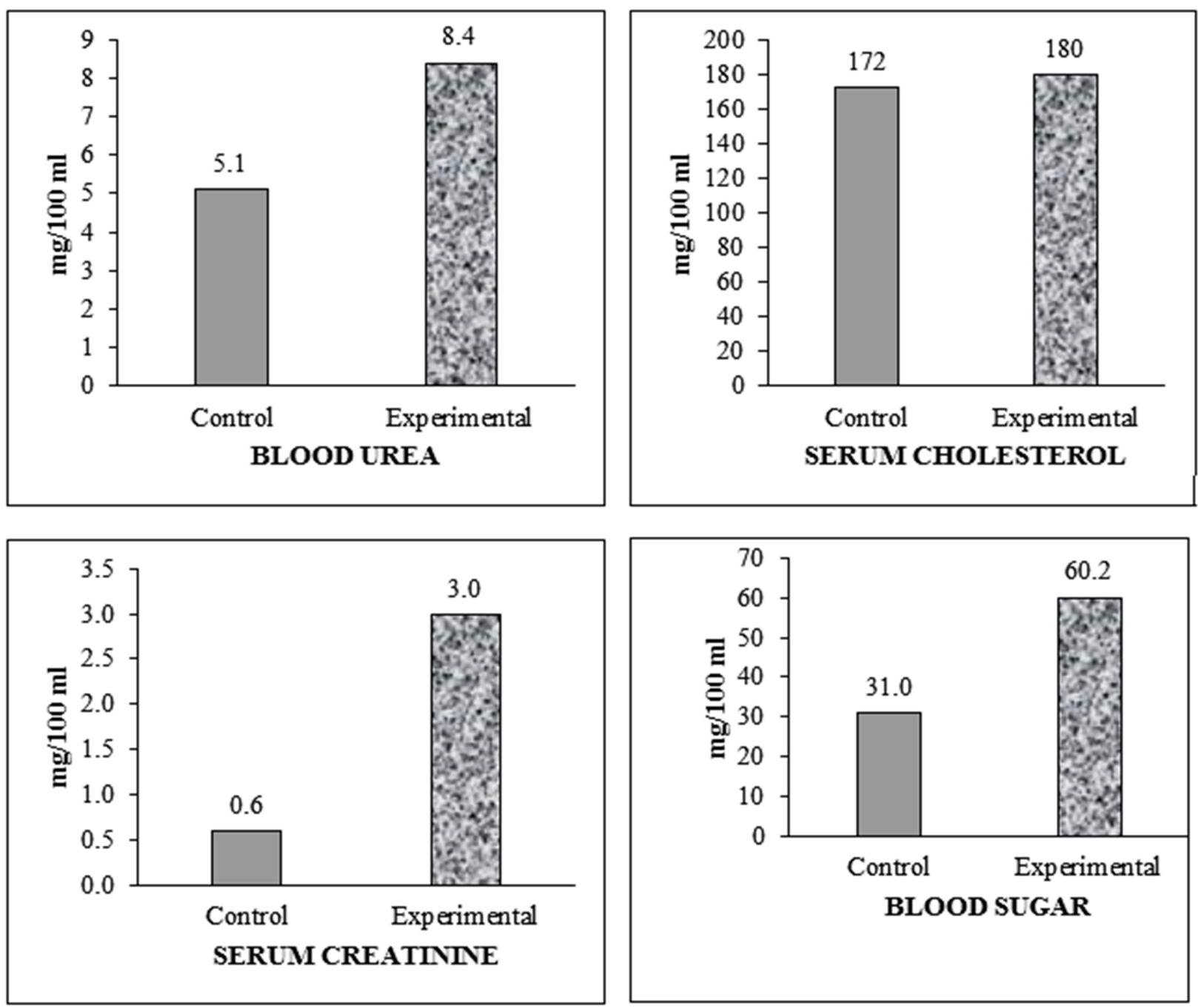

Figure 5. Bar diagrams of blood parameters after acute exposure to L-Lysine.

histopathological findings are supported by indicates that lysine does not interfere at a high hematological parameters such as highly significant degree with lipid metabolism even in a very high statistical increase in level of blood sugar from dose. Therapeutic use of a mixture of amino acids $31 \pm 2.13$ in control to $60.2 \pm 3.14 \mathrm{mg} / 100 \mathrm{ml}$ after in liver diseases shows their healing and acute exposure to high dose of L-lysine, it shows regenerative role. Many workers used Branched impaired sugar metabolism. Level of serum Chain Amino Acids (BCCAs) therapy in liver cholesterol is not increased significantly which cirrhosis. Clinical trials have suggested that BCCAs 
supplementation improves the prognosis of end stage liver failure reduced the mortality. cirrhotic patients and prevented hepatic failure (Muto et al., 2005). Some workers reported increased level of cholesterol in animals when fed on a high lysine dose. These findings of hypercholesterolemia are not in agreement with present study. Hepatotoxicity of lysine in mammals to this extent has not been reported.

Essential L-amino acids are used to treat renal disorders by many workers (Denis et al., 2016). The use of amino acids with Keto acids in the treatment of chronic renal failure was reported by Jungers and Chauveau (1988). All these workers applied essential amino acids along with Keto acids to treat the renal failure but no Nephrotoxicity of such supplements reported in chronic and acute treatments. The mixture of essential amino acids with Keto acids also used in treatment of liver failure. Recently the branched- chain amino acid therapy used in liver disorders (Charlton, 2006; Holecek, 2010; Tahira and Khan, 2015). These workers reported that use of these supplements in

\section{References}

Asanuma, K., Adochi, K., Sugimoto, T. and Chiba, S. 2006. Effects of lysine- induced acute renal failure in dogs. Journal of Toxicological Sciences, 87-98.

Bali and Singh, B.1994. Protective role of L-lysine amino acid in toxicity of Chromium in fish. Biosphere, 6(1): 19-24.

Charlton, M. 2006. Branched- chain amino acid enriched supplements as therapy for liver diseases. Journal of Nutrition, 136 (1 suppl) : 295S- 8S.

Crim, M. C. and Munro, H. N. 1977. Protein and amino acid requirements \& metabolism in relation to defined formula diets: In defined formula diets for medical purposes, ed: M.E. Shils pp. 5-15, American Medical Association, Chicago, IL.

Denis, F., Jing, C., Wei, C., Liliana, G., Hwang, S. J., Kamyar, K., Kopple, Z. J. D., Mitch, W. E. Giorgina, P., Vladimir, T. and Philippe, C. 2016. Adherence to keto acids/ essential amino acids- supplemented low protein diets and new indications for patients with chronic kidney diseases.

Dhami, B. S. 2012. Regenerative effect of L-lysine amino acid on gastric mucosa after chronic chromium toxicity. National seminar on changing scenario in life sciences for future challenges.

Holecek, M. 2010. Three targets of branched- chain amino acid supplementation in the treatment of liver disease. Nutrition, 26(5): 482-90.

\section{Conclusion}

All these findings show that the clinical use of essential amino acids is a new concept to treat the kidney and liver diseases but the toxicity of these supplements further warns their use in high doses. The present studies show that use of L-lysine amino acid in high doses is not safe and its further clinical trials are required to investigate because in this study it is found that lysine is much toxic in acute dose to vital organs like kidney and liver. The present studies show that essential amino acid Llysine caused nephrotoxicity and hepatotoxicity in experimental fish after acute treatment. In kidney it caused dilation of glomerular capillaries, glomerular haemorrhages and cloudy swelling of renal tubules with vacuolization. Vasodilation, and arteriosclerosis also observed in kidney, all these renal findings show toxicity of L-lysine in acute high dose.

John, F. B. 2018. Lysulin a new supplement for Nutritional support for People with Diabetes and Pre- diabetes (those at risk of developing diabetes). Diabetes Management, 3840 .

Jungers, P. and Chauveau, P. 1988. Amino acids and keto acids in the treatment of chronic renal failure. Blood Purification, 6(5): 299-314.

Lee, Da- Young and Kim, Eun-Hee 2019. Therapeutic Effects of Amino Acids in Liver Diseases, Current Studies and Future Perspectives. Journal of Cancer Prevention, 24(2): 72-78.

Malis, C. D., Recusen, L. C., Solez, K. and Whelton, A. 1984. Nephrotoxicity of lysine and of a single dose of aminoglycoside in rats given lysine. Journal of Laboratory and Clinical Medicine, 103: 660-676.

Meenu, S., Muralidhar, R, Shivansh, P., Sowjanya, B., Mahalakshmi, K., Dutt, K. R. and Ramesh, M. 2011. Medicinal uses of L-Lysine: Past and Future. International Journal of Research in Pharmaceutical science (IJRPS), 2(4): 637-642.

Munro, H. N. 1982. Metabolic integration of organs in health and diseases. J PEN.

Muto, Y., Sato, S., Watanabe, A., Moriwaki, H., Suzuki, K., Kato, A., Kato, M., Nakamura, T. and Nishiguchi, S. 2005. Effects of oral branched- chain amino acid granules on 
Dhami

event- free survival in patients with liver cirrhosis. Clinical Gastroenterology and Hepatology, 3: 705-713.

Park, J. G., Tak, W. Y. and Lee, W. K. 2017. Effects of branched chain amino acids (BCAAs) on the progression of advanced liver disease. Medicine (Baltimore), 96(24):e6580.

Recusen, L. C., Finn, W. F., Whelton, A. and Solez, K. 1985.Mechanism of lysine- induced acute renal failure in rats. Kidney International, 27: 517-522.

Samyuktha, P. and Francois, V. 2019. Dietary Amino Acids Affect The Rate of Chronic Kidney Disease Progression in Rats. FASEB, 33.S1.
Solez, K. 1983. Pathogenesis of acute renal failure. International review of experimental pathology, 24: 277333.

Steven, G., Munz, K.and Ulbricht, C. 2007. A Review of Dietary Supplement - Induced Renal Dysfunction. Clinical Journal of the American Society of Nephrology, 2: 757-765.

Tahira, S. and Khan, N. 2015. Nutrition as a part of therapy in treatment of liver cirrhosis. Journal of Nutrition \& Food Sciences, S11-004.

Venthan, J. M. and Sanketh, R. 2017. Lysine for Herpes Simplex Prophylaxis: A Review of the Evidence. Integrative medicine (Encinitas, Calif.) 16(3): 42-46. 